\title{
Contraintes de la traduction littéraire historiographique
}

\section{Basma Farid}

Pour chaque projet de traduction, le traducteur, à qui on fait appel, est amené à respecter un certain nombre de contraintes: délai, domaine de référence, type de supports, exigences clients... Autant de points qui sont à prendre en compte lors du travail et qui déterminent la qualité d'une traduction professionnelle.

La traduction n'est pas juste une affaire de langage. Pour obtenir un produit final de qualité et conforme aux attentes du client, le traducteur doit respecter et intégrer dans sa méthodologie un certain nombre d'exigences.

Notre point d'étude de cette recherche est basé sur l'ouvrage célèbre traduit de Khaled Mohamed Khaled (1920-1996), l'écrivain de Réjal Hawl El Rassoul $\left({ }^{1}\right)$. Cet ouvrage immense à la fois littéraire et historiographie était la cause de la réputation de Khaled.

Lorsque Khaled rédigeait son ouvrage, il voulait jeter la lumière sur les traces des compagnons durant la vie du Prophète (p.s.s.l) et surtout sur les éléments historiques. Il parvient à transmettre le côté humain des héros qui ont vécu avec le Prophète (p.s.s.l), qui ont participé à ses expéditions et qui sont morts pour la cause d'Allah et de son Messager (p.s.s.l).

Écrire la biographie de ces compagnons devient alors un moyen de transmettre un patrimoine islamique, historique et culturel unique et précieux dans notre monde où tout change si rapidement, affirme Shaker $\left({ }^{2}\right)$. Le terme « Historiographie » est employé donc pour désigner l'ensemble des ouvrages rédigés par les historiens à propos de leur objet d'étude $\left({ }^{3}\right)$.

Mounin voit que traduire, ce n'est pas une transposition dans une autre langue, c'est une invention. D'après lui, le traducteur est libre quant à la forme qu'il donne à cette invention, mais il est soumis à une double contrainte

- Détacher la lettre du sens commun.

- Respecter l'original, le garder dans son authenticité, viser en lui un langage pur, une écriture sacrée $\left({ }^{4}\right)$.

"Il doit produire le sens, rien que le sens. La langue d'arrivée doit être syntaxiquement et grammaticalement correcte, et le vocabulaire juste et précis, sans impropriété. Il faut respecter les niveaux de langue, tenir compte de la destination du texte, ajoute Mounin » $\left({ }^{5}\right)$.

Mais d'abord nous devons savoir quels sont les facteurs qui bloquent l'accès du lecteur au sens du texte source ? Quelle est la démarche des traducteurs dans ce cas ? Quelles sont les solutions adoptées et quels en sont les résultats? 
Certes, la traduction de notre texte, celle de l'Histoire islamique est une traduction spécialisée qui, en tant que telle, requiert, un travail maîtrisant les notions historiographiques fondamentales et les particularités de la science historique, ainsi que les stratégies et les difficultés de l'activité traductrice. Nous allons voir que les traducteurs de l'ouvrage ont essayé autant que possible de manipuler la langue de départ pour faciliter l'accès à la langue cible mais, parfois, faute de matériaux de travail consacrés à résoudre les problèmes spécifiques que pose la traduction, tels les dictionnaires monolingues et bilingues spécialisés, ils ont affronté pas mal de problèmes linguistiques. Prenons comme exemple l'expression arabe " أهل البيت qui figure ainsi dans le texte source :

" ذأك إمرؤ منا وإلينا أهل الييت ", nous pouvons observer une divergence de la traduction de cet énoncé rendu par «la famille de Mohamed» ( $1^{\text {ère }}$ traduction) et «La Maisonnée » ( ${ }^{\text {ème }}$ traduction). Les efforts des deux traducteurs s'avèrent clairs en cherchant le sens exact de cet énoncé à travers un dictionnaire monolingue.

" sont les personnes qui appartiennent à la famille du Prophète (p.s.s.l), ses épouses et ses filles $\left({ }^{6}\right)$ et «la Maisonnée » citée dans la deuxième traduction est l'ensemble de ceux qui habitent une même maison particulière $\left({ }^{7}\right)$. Par conséquent, les deux traducteurs nous offrent une bonne interprétation.

Au départ, nous allons analyser les contraintes socioculturelles qu'ont affrontées les traducteurs littéraires historiographes. Dans Les problèmes théoriques de la traduction, Mounin a montré que les langues peuvent poser des obstacles à la traduction $\left({ }^{8}\right)$.

Selon Danica Seleskovitch et Marianne Lederer, la traduction consiste à déverbaliser, après avoir compris, puis reformuler ou réexprimer.

"Il ne s'agit pas seulement de savoir quel mot placer dans

la langue d'arrivée en correspondance à celui de la langue de départ, mais aussi et surtout de savoir comment faire passer au maximum le monde implicite que recouvre le langage de l'autre " $\left({ }^{9}\right)$.

Il s'agit donc de la traduction de la culture ainsi que l'emploi des mots dits culturels. "Mot culturel » est le terme employé au lieu de «mots à référence culturelle spécifique ». Svane appelle les mots qui existent dans une culture et non pas dans une autre des « expressions référentielles » $\left({ }^{10}\right)$. Michel Ballard parle de " référents culturels » ou de " culturèmes », lesquels il définit comme un trait distinctif du texte d'arrivée. Tous ces termes visent à transmettre la même chose, à savoir les mots qui sont difficiles à traduire parce qu'ils dénotent des phénomènes qui n'existent que dans la langue de départ $\left({ }^{11}\right)$. 
Svane souligne que c'est important de se souvenir que le récepteur du texte ne reste pas le même après la traduction $\left({ }^{12}\right)$.C'est-à-dire que les mots culturels qui sont évidents pour le lecteur du texte original ne sont pas nécessairement évidents pour le lecteur du texte traduit parce que ce dernier ne partage pas la même culture que l'auteur. Par exemple, les deux termes « Mouhajirins et les Ansars » doivent être suivis d'une note explicative de la part des traducteurs pour désigner les Émigrés et les Médinois.

Codleanu parle de ce qu'on appelle zones de diversification. Elle explique que les systèmes linguistiques, dans lesquels évoluent les différentes communautés linguistiques, connaissent des zones de diversification spécifique mises en évidence lors de la traduction : ces zones concentrent des termes ou des séquences qui véhiculent une charge socio-culturelle spécifique $\left({ }^{13}\right)$. Par exemple, "صيام النافلة"c'est le jeûne bénévole des musulmans traduit par « le jeûne surérogatoire » dans le texte d'arrivée.

Il existe aussi des termes ou des séquences à charge civilisationnelle qui renvoient à des particularités locales :

-Coutumes arabes comme par exemple "الثِمِلة lou la cape, c'est aussi le vêtement de dessus, sans manches, qui enveloppe le corps et les bras porté par les Arabes $\left({ }^{14}\right)$.

-Croyances, culture matérielle.

-Des particularités géographiques comme par exemple"لبقيع El Baqi’ » ou les cimetières des Médinois.

-Systèmes socio-politiques comme "سوق الرقيق"traduit correctement par « le marché des esclaves $»$.

-Systèmes administratifs spécifiques ou qui renferment des allusions de toutes sortes: littéraires, historiques, folkloriques, etc ... $\left(\left(^{15}\right)\right.$

Ces séquences à charge civilisationnelle constituent plus qu'un problème de traduction dans le texte de Khaled. Codleanu voit que la traduction des séquences complexes figées englobant une allusion socioculturelle, comme nous venons de voir dans les exemples ci-dessous, peut imposer le recours à des traductions indirectes, le plus souvent, à une adaptation. Cette dernière est un ensemble de modalités de transfert culturel qui concernent les termes civilisationnels $\left({ }^{16}\right)$.

Dans le texte, Khaled utilise des expressions, des mots et des énoncés littéraires genre arabe classique qui posent déjà des problèmes de compréhension même pour un lecteur arabe. Prenons comme exemple, l'expression figée :

"qui s'attache seulement à l'environnement arabe. Cette expression veut dire que les bédouins, autrefois, comprenaient la nature de la personne dès qu'ils observaient son regard, son corps et la couleur de sa peau " فراسة البشر(القيافة)" Aussi, étaient-ils célèbres par la connaissance des 
traces des humains et des animaux "فراسة الأثر (العيافة) (17). Cette expression est loin d'être facile à comprendre, par conséquent sa traduction vers la langue cible demande un certain effort de la part des traducteurs qui doivent garder l'équilibre entre la compréhension du texte et sa déculturalisation, c'est-à-dire que lorsqu'ils suppriment les mots culturels, le texte ne reste pas implanté dans la culture originale. Ici dans cet exemple, les deux traducteurs ont adopté une stratégie qui se concentre sur la compréhension du texte. La $1^{\text {ère }}$ traduction a expliqué le sens de cette expression pour ne pas perdre la couleur locale du texte en disant: «les Arabes étaient les plus habiles à suivre les traces des pieds ». Cette adaptation pourrait être une bonne solution de sa part. Alors que la $2^{\text {ème }}$ traduction l'a complètement omise peut- être parce que le traducteur a vu que cette expression culturelle n'est pas essentielle au contexte.

Une autre allusion socio-culturelle figée existe dans l'expression : qui veut dire "تحت جوانحه" c'est une expression incomprise même dans son contexte $\left({ }^{19}\right)$. Les deux traducteurs ont négligé cette expression ce qui a rendu le texte d'arrivée obscur.

Aussi l'expression "فنلا ينبئك أنئذ مثل خبير" s'attache à la culture islamique et aux termes coraniques dans la sourate 35, verset 14. Elle veut dire que nul ne peut te donner des nouvelles comme Celui qui est parfaitement informé $\left({ }^{21}\right)$. La $1^{\text {ère }}$ traduction fait appel à l'une des formes de l'adaptation, à savoir, la périphrase explicative. Elle a rendu l'expression par «il l'examine et vous instruit alors comme un expert» ce qui est un bon choix. Tandis que la $2^{\text {ème }}$ traduction l'a complètement omise.

Ces énoncés ont besoin d'un lecteur cultivé pour comprendre les notions étrangères à la réalité française et un traducteur habile pour pouvoir transmettre leurs sens exacts sans défaillance ni faiblesse. Les traducteurs doivent sans cesse résoudre ces problèmes en trouvant des équivalences correctes. Pour ces différences culturelles, les traducteurs ont eu recours à l'adaptation afin que le lecteur français ne sente pas qu'il s'agit d'une traduction.

D'autre part, nous pouvons dire que les noms propres des personnes ont un grand pouvoir d'évocation en dehors de leur fonction dénominative. Ils peuvent créer des associations d'esprit et des images. Charolles voit que ces noms ont un fonctionnement référentiel mais peuvent également être accompagnés d'une série de connotations et renferment des allusions difficilement transmissibles en langue cible. En certaines situations « le nom propre peut perdre sa valeur dénominative pour prendre une valeur qualificative » $\left({ }^{22}\right)$.Par exemple le nom de famille de Salman qui est «El Farressy », est ambigu pour un lecteur non familier avec la langue arabe. Ce lecteur ne comprend pas que Salman est persan et il est appelé « Faressy » pour connoter son pays d'origine, la Perse. 
L'utilisation de la lettre majuscule du nom propre est aussi une aide pour le traducteur puisque le lecteur comprend, par exemple, qu'il est question d'une ville ou d'un nom de compagnon. Mais comme on ne peut pas s'attendre à ce que le lecteur puisse savoir que le mot bani en arabe (بنى )signifie tribu en français comme Banni Assad, Banni Zouhra, c'est important que les deux traducteurs ajoutent une précision ou une certaine note explicative.

Ballard écrit dans Le nom propre en traduction que le traducteur doit se demander pourquoi un nom propre est utilisé. Il écrit qu'il y a deux types d'exploitation du nom propre :

" [l]'un relève de la fonction de décor, d'indication scénique » et "l'autre est une exploitation plus directe et ponctuelle où apparaît de façon plus évidente et visible l'intervention du narrateur, généralement sous la forme d'un commentaire» $\left({ }^{23}\right)$.

Les noms propres arabes mentionnés dans les deux versions sont directement traduits. Le nom de ces tribus n'a causé aucun problème aux deux traducteurs ce qui aide à préserver la couleur locale.

Cependant, nous remarquons que le $2^{\text {ème }}$ traducteur utilise la majuscule dans toutes les lettres du surnom «Mouss'ab ALKHAIR》 ou en se référant au compagnon charitable Mouss'ab Ibn Omayr, afin d'attirer l'attention du lecteur sur sa personnalité généreuse. Ballard voit que c'est une stratégie qu'on peut utiliser pour traduire les mots culturels surtout que le contexte ne fournit pas au lecteur l'information nécessaire pour qu'il comprenne de quoi il s'agit $\left({ }^{24}\right)$.

Quant au toponymes, Ballard voit qu'on ne les traduit pas généralement. Il ajoute que la question de savoir si on traduit le nom d'un toponyme international ou non dépend de l'usage $\left({ }^{25}\right)$ comme par exemple qui figure dans le texte arabe traduit correctement par Abyssinie . Nous soulignons que presque toutes les mentions de toponymes arabes ne causent aucun problème de compréhension dans les deux versions comme par exemple : Taef, La Mecque, l'Éthiopie, la Médine, la Perse, Sanaa, la Syrie, l'Egypte et l'Iraq.

Cependant, les deux traducteurs font précéder le nom des lieux des combats par le terme (jour) pour montrer au lecteur non arabophone qu'il s'agit d'une bataille historique comme par exemple : le jour d'Ohoud, le jour de Qarbala', le jour de Tabouq, le jour de Khaybar ...etc. Ils essaient de transmettre ces connotations qui réfèrent à un événement historique à travers une traduction directe pour que le texte soit homogène. Mais cette traduction ne fonctionne pas bien parce que le lecteur français ne comprend pas qu'il est question d'un combat. Alors ils auraient dû traduire ce terme par bataille ou combat par exemple pour faciliter une bonne assimilation du mot. 
On peut donc conclure que les allusions de nature socio-culturelle spécifique posent aux traducteurs des problèmes de transfert dans une autre culture sauf s'ils sont très prudents dans leurs versions. Le fait qu'un traducteur possède la même culture que l'auteur facilite beaucoup sa tâche. La structure qui contient l'allusion socio-culturelle renferme une charge de vocabulaire très riche et rend parfois difficile l'accès au sens du message du texte d'arrivée. C'est pour cela, être un bon traducteur, il faut avant tout être un bon rédacteur dans sa propre langue, mais à condition de mettre son talent au service du message de l'auteur. Le traducteur n'est pas un créateur, mais un recréateur comme l'affirme Delisle $\left({ }^{26}\right)$.

\section{Sources et bibliographie}

- BALLARD, Michel, Le nom propre en traduction, Paris, Gap, 2001

- _ «Le cultèreme en traduction : entre sens indice et écriture », in Eriksson, 18 novembre 2007, Vajio University Press

- CHAROLLES, Michel, La référence et les expressions référentielles en français, Paris, Ophrys, 2002

- CODLEANU, Mioara, "Interculturalité et intercommunication »,In Signes et discours, no. 15, juin 2008

- COMPLEXE DU ROI FAHD, Le Noble Coran et la traduction en langue française de ses sens, Al Madinah Mounawarah, Presses du complexe du roi Fahd,1410 de l'Hégire

- CRISTEA,Téodora, Stratégies de la traduction, La Roumanie, Ed. Fundatiei, 2000

- DELISLE, Jean, La traduction en citations, Canada, Les Presses de l'Université d'Ottawa, 2007

- EL NABOULSSY, Shaker, la révolution du patrimoine : étude de la pensée de Khaled Mohamed Khaled, Le Caire, Dar El Nahda, 1955

- FONTANIER, Pierre, Les Figures du discours, Paris, Flammarion, 1977.

- IBN MANZOUR, Mo'ğam Lessan El 'Arabe, Beyrouth, Dar El Qotob, 1970

- KHALED, Mohamed, Réjal Hawl El Rassoul, Liban, Dar El Fikr, 2002 , Des hommes autour de l'Envoyé de Dieu, traduction de Rima Ismail, Liban, Dar El Fiqr, 2002.

- $\quad$ Des hommes autour du Prophète, traduction de Abdou Harkat, Liban, Dar el Kotob el 'Elmeya, 2005 
- MOUNIN, Georges, Les problèmes théoriques de la traduction, Paris, Gallimard, 1963

- REIG, Daniel, Dictionnaire arabe Français, Paris, Larousse, 2001

- REY, Alain, Le Grand Robert de la langue française, version électronique, 2017

- SELESKOVITCH, Danica et LEDERER, Marianne, Interpréter pour traduire, Paris, Didier, 1983

- SVANE, B, «Comment traduire la réalité ? Étude de la traduction des expressions référentielles » in Eriksson, 1998

${ }^{1}$ Khaled, Mohamed, Réjal Hawl El Rassoul, Liban, Dar El Fikr, 2002

${ }^{2}$ El Naboulssy, Shaker, la révolution du patrimoine: étude de la pensée de Khaled Mohamed Khaled, Le Caire, Dar El Nahda, 1955, p.15

${ }^{3}$ Svane, B, «Comment traduire la réalité ? Étude de la traduction des expressions référentielles » in Eriksson, 1998, p. 93

${ }^{4}$ Mounin, Georges, Les problèmes théoriques de la traduction, Paris, Gallimard, 1963, p.37

${ }^{5}$ Id, Ibid.

أهل البيت هم 2249 أزواج النبى وبناته .

${ }^{7}$ Rey, Alain, Le Grand Robert de la langue française, version électronique, 2017

${ }^{8}$ Mounin, Op.cit., p.122

${ }^{9}$ Seleskovitch, Danica et Lederer, Marianne, Interpréter pour traduire, Paris, Didier, 1983, p.98

${ }^{10}$ Svane, B, Op.cit., pp. 93-118

${ }^{11}$ Ballard, Michel, "Le cultèreme en traduction : entre sens indice et écriture », in Eriksson, 18 novembre 2007, Vajio University Press,pp. 19-40

12 Svane, B. Op.cit., p. 110

${ }^{13}$ Codleanu, Mioara, « Interculturalité et intercommunication »,In Signes et discours, no.15, juin 2008, p.23

${ }^{14}$ Reig, Daniel, Dictionnaire arabe Français, Paris, Larousse, 2001, p.2957

${ }^{15}$ Codleanu, Op.cit.,

${ }^{16}$ Cristea,Teodora, Stratégies de la traduction, La Roumanie, Ed. Fundatiei, 2000, p.111

${ }^{17}$ Ibn Manzour, Op.cit., p. 3776 et 3192

${ }^{18} \mathrm{Ibid}, \mathrm{p} .697$

${ }^{19}$ Khaled, Réjal Hawl El Rassoul, Op.cit., p.11 فيدرك ما تحت جو انجه من صدق وبهنتان

${ }^{20}$ Ibid.,

${ }^{21}$ Complexe du Roi Fahd, Le Noble Coran et la traduction en langue française de ses sens, Al Madinah Al Mounawarah, Presses du complexe du roi Fahd,1410 de l'Hégire, s.35, v.14

${ }^{22}$ Charolles, Michel, La référence et les expressions référentielles en français, Paris, Ophrys, 2002, p. 25

${ }^{23}$ Ballard, Michel, Le nom propre en traduction, Paris, Gap, 2001, p.124

${ }^{24}$ Id, Ibid.,p. 126

${ }^{25}$ Id, Ibid, p. 132

${ }^{26}$ Delisle, Jean, La traduction en citations, Canada, Les Presses de l'Université d'Ottawa, 2007,p.53 